\title{
The Prognostic Importance of Microsatellite Instability Status in Turkish Stage II and III Gastric Cancer Patients who Received Adjuvant Chemotherapy
}

\author{
Serdar ARICI ${ }^{1}$, Selma Sengiz ERHAN ${ }^{2}$, Caglayan GEREDELI ${ }^{1}$, Muhammed Mustafa ATCI ${ }^{1}$, \\ Saban SECMELER ${ }^{1}$, Ruhper CEKIN ${ }^{1}$, Abdullah SAKIN ${ }^{3}$, Sener CIHAN ${ }^{1}$ \\ ${ }^{1}$ University of Health Sciences, Okmeydani Training and Research Hospital, Department of Medical Oncology, Istanbul, \\ ${ }^{2}$ University of Health Sciences, Okmeydani Training and Research Hospital, Department of Pathology, Istanbul \\ ${ }^{3}$ Yuzuncu Yil University Faculty of Medicine, Department of Medical Oncology, Van, TURKEY
}

\begin{abstract}
Some retrospective studies in different populations have evaluated the prognostic value of microsatellite instability status (MSI) in patients with gastric cancer (GC). A small number of studies have focused on the effect of MSI status on the outcome of GC patients who have received adjuvant chemotherapy (CT). Medical records of 318 patients with stage II or III GC who had been treated with adjuvant CT after D2 gastrectomy between 2016 and 2019 were scanned. Eligible patients were divided into two groups: MSI-H and microsatellite stable (MSS). The determinant factors were examined using Cox regression analysis. A statistical significance level of alpha was accepted as $p<0.05$. The study included 207 GC patients and 21 (10.1\%) MSI-high patients. A median disease-free survival was not reached (95\% CI NR) in MSI-H patients, whereas a median disease-free survival was 30 months in MSS patients (95\% Cl 24.3-35.6) ( $p=0.046)$. A median overall survival (OS) was not reached in MSI-H patients, whereas a median overall survival of 46 months (95\% Cl: 28.8 - 60.1) was reached in MSS patients ( $p=0.032)$. In the multivariate Cox regression analysis for OS, female gender and MSI-H status were positive predictors of OS, whereas stage III disease negatively affected OS $(p=0.009, p=0.030$, and $\mathrm{p}=0.009$, respectively). Microsatellite instability status may be a prognostic factor in stage II and III Turkish GC patients who have received adjuvant oxaliplatin-based $\mathrm{CT}$.
\end{abstract}

Keywords: Microsatellite instability, Gastric cancer, Adjuvant chemotherapy

\section{INTRODUCTION}

Gastric cancer (GC) is one of the leading causes of cancer-related deaths worldwide. ${ }^{1}$ Surgery is the main treatment for patients with localized GC, although a large number develop recurrence or metastasis even after curative resection. Recent randomized trials and meta-analyses have indicated a significant survival improvement over surgery alone for a number of approaches, including adjuvant chemoradiotherapy, perioperative chemotherapy (CT), and adjuvant $\mathrm{CT}$ alone for patients with potentially resectable GC. ${ }^{2,3}$ However, there is no validated biomarker to determine outcomes in GC patients who have received adjuvant $\mathrm{CT}{ }^{4}$
In colorectal cancer, microsatellite instability (MSI) testing is routinely performed in stage II CRC patients to guide adjuvant treatment decisions, since MSI-high (MSI-H) status is associated with significantly better stage-specific survival and a possible lack of efficacy of adjuvant 5-fluorouracil (FU). In recent years, four molecular subtypes of GC have been identified via molecular classification based on next generation sequencing data: Epstein-Barr virus-positive subtype, MSI subtype, genomically stable subtype, and chromosomally unstable subtype..$^{5}$ Although highly variable, the frequency of MSI-H is approximately $10 \%$ in GC clinical studies. ${ }^{6,7}$ 
Some retrospective studies have evaluated the prognostic value of MSI in patients with GC. ${ }^{8,9}$ The biological reason for better survival rates is attributed to the association between MSI-H and other molecular features, leading to increased hostrelated immune responses, such as programmed death-ligand $1 .{ }^{10}$ However, studies have shown a potentially detrimental or null effect of MSI-H for perioperative $\mathrm{CT}$ in resected GC. ${ }^{11} \mathrm{~A}$ small number of studies have focused on the effect of MSI status on the outcome of GC patients who have received adjuvant $\mathrm{CT}$.

Therefore, we aimed to investigate whether MSI status affects disease-free survival (DFS) and overall survival (OS) in stage II-III Turkish GC patients who have received adjuvant chemotherapy.

\section{PATIENTS AND METHODS}

\section{Study Population}

The medical records of 318 patients with stage II or III GC who had been treated with adjuvant CT after D2 gastrectomy between 2016 and 2019 in the medical oncology clinic at University of Health Sciences, Okmeydani Training and Research Hospital were scanned. The staging procedure of the patients was performed according to the pathology reports. The following parameters were defined as the exclusion criteria: age $<18$ years; patients with a second primary malignancy; patients with a benign or malign hematological disorder; patients with evidence of metastasis at diagnosis; patients with missing data; patients who had surgery other than D2 gastrectomy; and patients whose MSI status could not be evaluated. Eligible patients were divided into two groups: MSI-H and microsatellite stable (MSS) status.

In the adjuvant setting, patients had received 21day cycles of capecitabine $\left(1000 \mathrm{mg} / \mathrm{m}^{2}\right.$ twice daily on days 1 to 14$)$ plus oxaliplatin $\left(130 \mathrm{mg} / \mathrm{m}^{2}\right.$ on day 1) (CAPOX) or 14-day cycles of oxaliplatin $\left(85 \mathrm{mg} / \mathrm{m}^{2}\right.$ on day 1) plus infusional FU $(400 \mathrm{mg} /$ $\mathrm{mg} / \mathrm{m}^{2}$ on day 1 and $2400 \mathrm{mg} / \mathrm{m}^{2}$ on day 1-2) and leucovorin $\left(400 \mathrm{mg} / \mathrm{m}^{2}\right.$ on day 1 ) (FOLFOX).

\section{Data Collection}

Demographic and disease characteristics, such as age, gender, Eastern Cooperative Oncology Group
(ECOG) status, date of diagnosis, histology (adenocarcinoma or signet ring histology and Lauren histotype: intestinal or diffuse type), grade, stage, human epidermal growth factor receptor 2 (HER 2) status, tumor location, baseline carcinoembryonic antigen (CEA), carbohydrate antigen 19-9 (CA 19-9), CT regimens (CAPOX or FOLFOX), recurrence date, last status (death or alive), and MSI-H or MSS status were obtained from archived patient medical files. Disease staging was performed according to the Tumor, Node, Metastasis (TNM) staging system (eighth edition).

\section{Microsatellite Instability Status Assessment}

A formalin-fixed paraffin-embedded block was selected for each case, which exemplified the invasive areas of the tumor. Paraffin-embedded tissue blocks were cut into $3 \mu \mathrm{m}$ sections. IHC was performed using a Ventana XT automated stainer (Ventana Corp., Tucson, AZ, USA) with antibodies to MLH1 (ready-to-use, clone ES05 DAKO, Denmark), MSH2 (ready-to-use, clone FE11 DAKO, Denmark), MSH6 (ready-to-use, clone EP49 DAKO, Denmark), and PMS2 (ready-to-use, clone EP51 DAKO, Denmark). Sections were deparaffinized using EZ Prep solution (Ventana Corp.). The CC1 standard (pH 8.4 buffer containing Tris/ borate/EDTA) was used for antigen retrieval and blocked with inhibitor $\mathrm{D}\left(3 \% \mathrm{H}_{2} \mathrm{O}_{2}\right)$ for 4 minutes at $37^{\circ} \mathrm{C}$. Slides were incubated with the primary antibody for 40 minutes at $37^{\circ} \mathrm{C}$, followed by a universal secondary antibody for 20 minutes at $37^{\circ} \mathrm{C}$. Slides were incubated in streptavidin-horseradish peroxidase (SA-HRP) D for 16 minutes at $37^{\circ} \mathrm{C}$. Then, the substrate, 3,3'-diaminobenzidine tetrahydrochloride (DAB) $\mathrm{H}_{2} \mathrm{O}_{2}$, was added for 8 minutes, followed by hematoxylin and bluing reagent counterstaining at $37^{\circ} \mathrm{C}$. A loss of MMR (mismatch repair) protein expression was designated when none of the neoplastic epithelial cells showed nuclear staining, while normal expression was defined as the presence of nuclear staining of tumor cells, irrespective of the proportion or intensity. Infiltrating lymphocytes, stromal cells, and adjacent nonneoplastic epithelium served as internal positive controls. While normal expression was considered MSS, at least one of four antibodies was considered MSI-H. ${ }^{12,13}$ 
International Journal of Hematology and Oncology

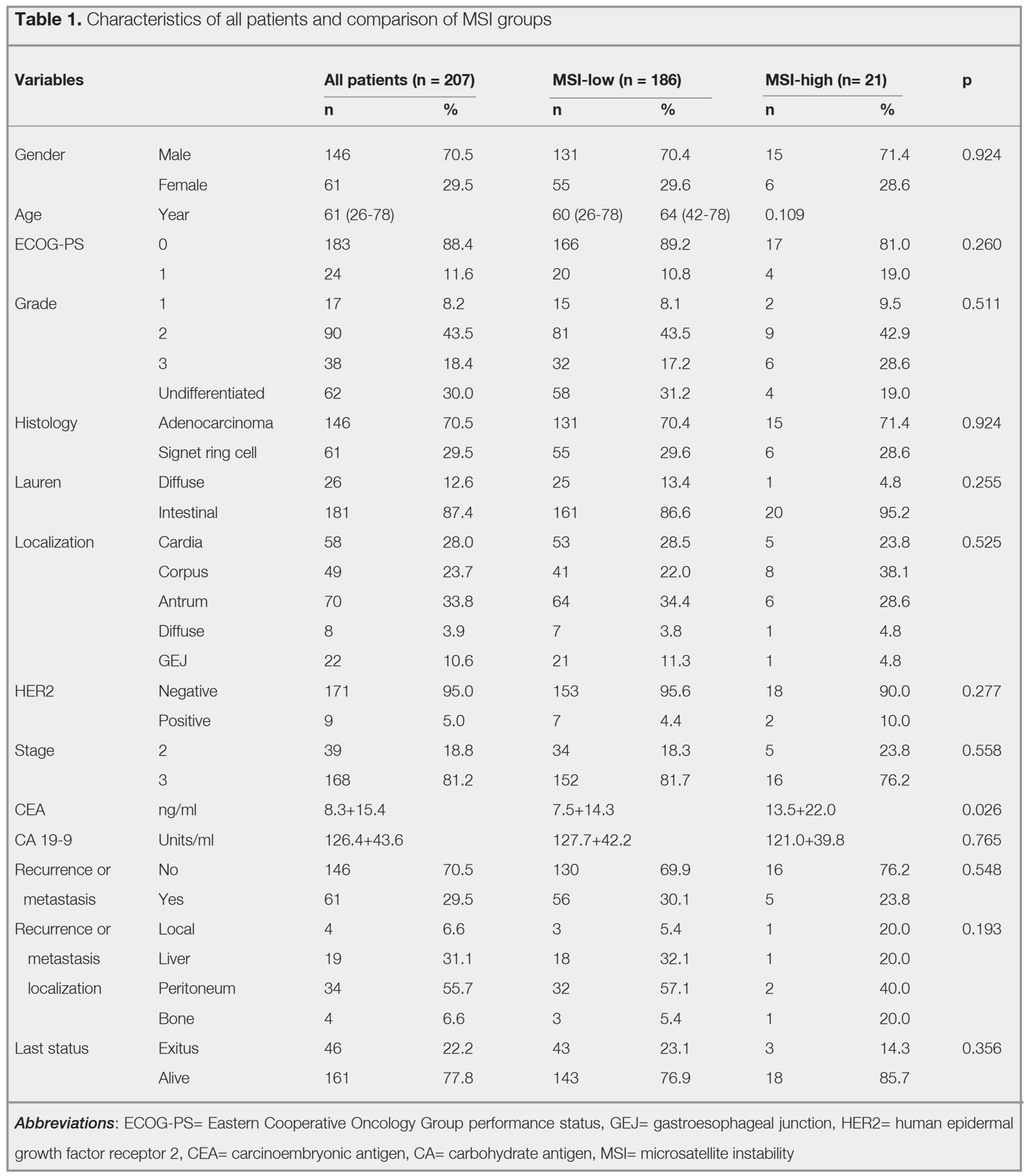

The study was performed in accordance with the Declaration of Helsinki. The patients gave written informed consent before the study. Both patient consent and approval by the University, University of Health Sciences, Okmeydani Training and Research Hospital Ethics Committe (16.06.2020/ 48670771-514.10) were received.

\section{Statistical Analysis}

SPSS 15.0 for Windows was used for statistical analysis. Descriptive statistics were given as a number and a percentage for categorical variables, average, and standard deviation, and as a minimum and a maximum for numeric variables. Comparisons of the numerical variables in two independ- 


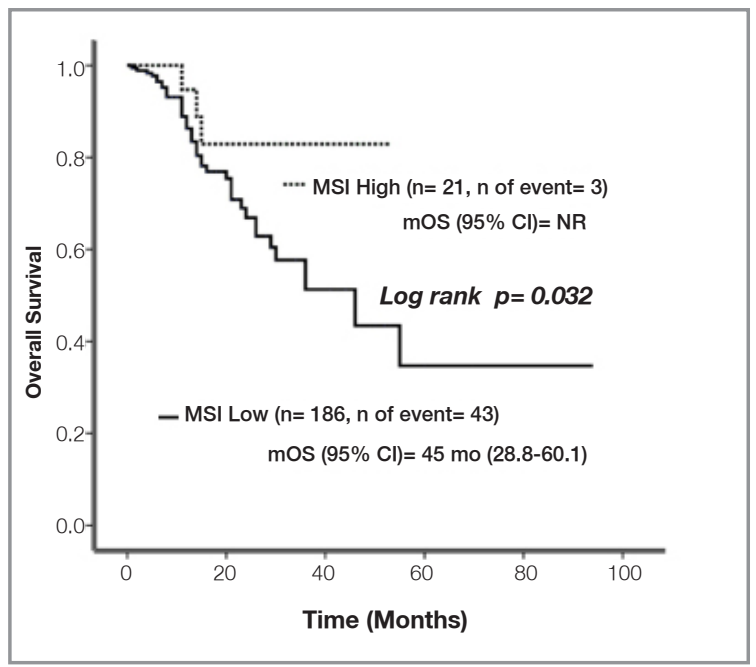

Figure 1. Disease-free survivals of MSI-high and MSI-low patients

ent groups were made using the Mann-Whitney $\mathrm{U}$ test since the normal distribution condition was not met. Comparisons of the ratios in the groups were made using the chi-square test. The determinant factors were examined using Cox regression analysis. The Kaplan-Meier estimate was used to estimate the survival function, and a statistical significance level of alpha was accepted as $p<0.05$.

\section{RESULTS}

\section{Patient Characteristics}

The study included 207 GC patients, consisting of $146(70.5 \%)$ males and $61(29.5 \%)$ females. The median age was 61 years (range: 26-78 years). Of the 207 patients, 183 (88.4\%) had ECOG-PS 0 and $24(11.6 \%)$ had ECOG-PS 1 . The number of patients with intestinal and diffuse GC was $181(87.4 \%)$ and $26(12.6 \%)$, respectively. Sixtyone $(29.5 \%)$ patients had a signet ring histology component. One-hundred patients (48.4\%) had a poorly differentiated or undifferentiated tumor. There were nine $(5.0 \%)$ HER2-positive patients. Cardia tumors were seen in $58(28.0 \%)$ patients. There were $39(18.8 \%)$ and $168(81.2 \%)$ stage II and III patients, respectively. The mean CEA level was $8.3 \pm 15.4 \mathrm{ng} / \mathrm{ml}$, and the mean CA 19-9 level was $126.4 \pm 43.6$ units $/ \mathrm{ml}$. Recurrence or distant metastasis occurred in 61 patients $(29.5 \%)$. The

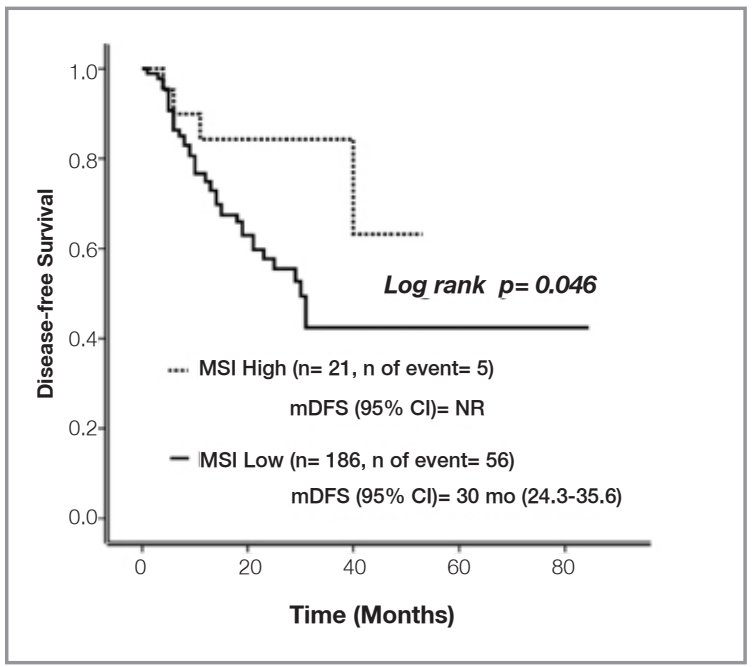

Figure 2. Overall survivals of MSI-high and MSI-low patients

most common metastasis sites were the peritoneum, liver, and bone. Forty-six (22.2\%) patients had died (see Table 1).

There were $21(10.1 \%)$ MSI-H patients and 186 (89.9\%) MSS patients. When the MSI-H and MSS patients were compared, the only difference was seen in CEA levels. The mean CEA levels were $13.5 \pm 22.0 \mathrm{ng} / \mathrm{ml}$ and $7.5 \pm 14.3 \mathrm{ng} / \mathrm{ml}$ in the MSI$\mathrm{H}$ and MSS patients, respectively $(\mathrm{p}=0.026)$ (see Table 1).

\section{Outcomes}

In the follow-up period, a median DFS was not reached (95\% CI NR) in MSI-H patients, whereas a median DFS of 30 months was reached in MSS patients (95\% CI: 24.3-35.6). This difference was significant $(\mathrm{p}=0.046)$ (Figure 1). A median OS was not reached in MSI-H patients, whereas a median OS of 46.0 months (95\% CI 28.8-60.1) was reached in MSS patients $(\mathrm{p}=0.032)$ (Figure 2$)$.

In the univariate Cox regression analysis for OS, female gender and MSI-H status were associated with favorable OS $(\mathrm{p}=0.008, \mathrm{p}=0.033$, respectively), whereas signet ring histology and stage III disease negatively affected OS $(\mathrm{p}=0.038, \mathrm{p}=$ 0.011 , respectively). Likewise, in the multivariate Cox regression analysis for OS, female gender and MSI-H status were positive predictors of OS $(\mathrm{p}=$ 
International Journal of Hematology and Oncology

\begin{tabular}{|c|c|c|c|c|c|c|c|}
\hline \multirow[t]{2}{*}{ Variables } & & \multicolumn{3}{|c|}{ Univariate Analysis for OS } & \multicolumn{3}{|c|}{ Multivariate Analysis for OS } \\
\hline & & HR & $95 \% \mathrm{Cl}$ for $\mathrm{HR}$ & $\mathbf{p}$ & HR & $95 \% \mathrm{Cl}$ for $\mathrm{HR}$ & $\mathbf{p}$ \\
\hline Age & Year & 1.011 & $0.979-1.045$ & 0.503 & & & \\
\hline Gender & Female vs male & 0.287 & $0.113-0.726$ & 0.008 & 0.292 & $0.115-0.740$ & 0.009 \\
\hline ECOG-PS & 1 vs 0 & 1.748 & $0.808-3.778$ & 0.156 & & & \\
\hline \multirow[t]{4}{*}{ Grade } & 1 (ref) & & & 0.463 & & & \\
\hline & 2 & 0.894 & $0.297-2.688$ & 0.842 & & & \\
\hline & 3 & 0.950 & $0.305-2.959$ & 0.929 & & & \\
\hline & Undifferentiated & 1.577 & $0.511-4.862$ & 0.428 & & & \\
\hline Histology & Signet ring cell & 1.861 & $1.034-3.350$ & 0.038 & & & \\
\hline Lauren & Intestinal vs diffuse & 0.958 & $0.404-2.274$ & 0.923 & & & \\
\hline \multirow[t]{5}{*}{ Localization } & Cardia (ref) & 1 & & 0.743 & & & \\
\hline & Corpus & 1.23 & $0.573-2.635$ & 0.595 & & & \\
\hline & Antrum & 0.748 & $0.329-1.697$ & 0.488 & & & \\
\hline & Diffuse & 1.351 & $0.298-0.696$ & 0.696 & & & \\
\hline & GEJ & 1.252 & $0.464-3.374$ & 0.656 & & & \\
\hline HER2 & Positive vs negative & 1.015 & $0.244-4.212$ & 0.984 & & & \\
\hline $\mathrm{MSI}$ & High vs low & 0.299 & $0.092-0.973$ & 0.033 & 0.290 & $0.089-0.946$ & 0.030 \\
\hline Stage & III vs II & 3.840 & $1.363-10.812$ & 0.011 & 3.948 & $1.404-11.103$ & 0.009 \\
\hline CEA & $\mathrm{ng} / \mathrm{ml}$ & 1.008 & $0.995-1.023$ & 0.234 & & & \\
\hline CA 19-9 & Units/ml & 1.000 & $0.999-1.001$ & 0.721 & & & \\
\hline
\end{tabular}

$0.009, p=0.030$, respectively), whereas stage III disease negatively affected OS $(p=0.009$, respectively) (Table 2).

\section{DISCUSSION}

In this study, we investigated whether MSI status affected the DFS and OS in stage II-III GC patients who had received oxaliplatin-based adjuvant chemotherapy after D2 gastrectomy. We found that the MSI-H patients' ratio was $10.1 \%$, and better OS and DFS were seen in MSI-H GC patients.

Some studies have suggested that MSI status in GC patients is associated with geographical background. In one study, the frequency of MSI-H in Japanese versus European American GC specimens was investigated and found to be $39 \%$ versus $20 \%$, respectively. Different MSI-H rates were also detected in different European regions in the same study. ${ }^{14}$ In another study, the MSI-H group was found to constitute $7.5 \%$ of German GC patients. ${ }^{15}$
Buffart et al. ${ }^{16}$ looked at genomic instability patterns of GC obtained from Caucasian and South African patients and observed that Caucasian patients showed significantly more MSI-H tumors. In an Italian study, differences in MSI status between GC patients from high-risk and low-risk areas of Italy were evaluated; MSI-H was observed in $23.9 \%$ of all GC patients studied. Patients from high-risk areas showed a higher rate of MSI-H tumors. ${ }^{17}$ In the present study, we found a rate of $10.1 \%$ for MSI-H tumors in stage II and III Turkish GC patients.

It was also observed that MSI-H status was associated with different factors, such as tumor Lauren histotype, grade, stage, female gender, and age (older patients) in different populations. ${ }^{18-20} \mathrm{We}$ found higher CEA levels in MSI-H patients than in MSS patients. Similarly, in a study of colorectal cancer patients, MSI-H tumors were associated with a high level of baseline CEA. ${ }^{21}$ Other factors were similar in the two groups in our study. 
Some studies have focused on prognosis based on MSI status in GC patients, with different results. Kim et al. ${ }^{22}$ evaluated the outcomes of microsatellite instability in early GC and found that the prognosis of MSI-H tumors was similar to that of MSS tumors. However, this trial included only mucosal or submucosal GC patients. In the post hoc analysis of the ARTIST trial, including radically resected GC patients randomized to adjuvant capecitabine/ cisplatin alone or combined with radiotherapy, the MSI prognostic effect on DFS and OS was evaluated. There was a trend toward a better but not significant difference in MSI-H patients compared with MSS patients in terms of DFS and OS. ${ }^{23}$ However, in the Italian study discussed above, it was observed that the 5-year survival for MSS patients from high- and low-risk areas was 32.9\% and 36\%, respectively. For the MSI-H patient group, the 5 -year survival was $67.3 \%$ for high-risk areas and $76.9 \%$ for low-risk areas. ${ }^{17}$ However, in this study, the number of patients who had received adjuvant CT was very low.

In comparison, the post hoc analysis of the ITACA-S trial, which included 256 patients, showed that MSI-H status was an independent favorable factor for both DFS and OS in stage II and III GC patients who had received adjuvant or perioperative CT. ${ }^{24}$ In the post hoc analysis of the CLASSIC trial, which included patients who had been treated with D2 gastrectomy alone or adjuvant CT after D2 gastrectomy, 19 (6.2\%) MSI-H patients had received adjuvant CT; 21 had received surgery alone. Disease-free survival was evaluated in groups, and MSI-H was an independent factor for DFS. In addition, adjuvant CT was associated with improved DFS in the MSS group but not in the MSI-H group. Overall survival was not analyzed according to MSI status. ${ }^{25}$ In the present study, we analyzed stage II and III patients who underwent D2 gastrectomy and adjuvant oxaliplatin-based CT and found that both DFS and OS were improved in MSI-H patients.

Our trial has a few limitations. First, this study was planned retrospectively, which might lead to several biases. In addition, it did not include any patients who underwent D2 gastrectomy without adjuvant CT. However, as illustrated by the studies discussed above, the effects of MSI-H on progno- sis have been shown to differ by patient's ethnicity. For this reason, our study is important because it is the first study to show the prognostic role of MSI status on stage II and III GC in Turkish patients. In addition, we evaluated OS in terms of MSI status in patients who had undergone $\mathrm{D} 2$ gastrectomy and received adjuvant $\mathrm{CT}$.

\section{CONCLUSION}

Microsatellite instability status may be a prognostic factor for stage II and III Turkish GC patients who have received adjuvant oxaliplatin-based CT. Also, improved OS of MSI-H patients may suggest that MSI status is useful as a predictive marker in adjuvant settings. These results need to be confirmed by randomized trials.

\section{REFERENCES}

1. Bray F, Ferlay J, Soerjomataram I, et al. Global cancer statistics 2018: GLOBOCAN estimates of incidence and mortality worldwide for 36 cancers in 185 countries. CA Cancer J Clin 68: 394-424, 2018.

2. Smyth EC, Verheij M, Allum W, et al. Gastric cancer: ESMO Clinical Practice Guidelines for diagnosis, treatment and follow-up. Ann Oncol 27: v38-v49, 2016.

3. Sakin A, Aydemir O, Sahin S, et al. Red blood cell distribution width as a possible predictor of diagnosis and survival in gastric cancer. EJMl 4: 289-297, 2020.

4. Corso S, Giordano S. How can gastric cancer molecular profiling guide future therapies? Trends Mol Med 22: 534-544, 2016.

5. Cancer Genome Atlas Research N. Comprehensive molecular characterization of gastric adenocarcinoma. Nature 513: 202-209, 2014.

6. Lee J, Lim DH, Kim S, et al. Phase III trial comparing capecitabine plus cisplatin versus capecitabine plus cisplatin with concurrent capecitabine radiotherapy in completely resected gastric cancer with D2 lymph node dissection: the ARTIST trial. J Clin Oncol 30: 268-273, 2012.

7. Bang YJ, Kim YW, Yang HK, et al. Adjuvant capecitabine and oxaliplatin for gastric cancer after D2 gastrectomy (CLASSIC): a phase 3 open-label, randomised controlled trial. Lancet 379: 315-321, 2012.

8. Marrelli D, Polom K, Pascale V, et al. Strong Prognostic Value of Microsatellite Instability in Intestinal Type Non-cardia Gastric Cancer. Ann Surg Oncol 23: 943-950, 2016. 
9. Polom K, Marano L, Marrelli D, et al. Meta-analysis of microsatellite instability in relation to clinicopathological characteristics and overall survival in gastric cancer. $\mathrm{Br} \mathrm{J}$ Surg 105: 159-167, 2018.

10. Llosa NJ, Cruise M, Tam A, et al. The vigorous immune microenvironment of microsatellite instable colon cancer is balanced by multiple counter-inhibitory checkpoints. Cancer Discov 5: 43-51, 2015.

11. Smyth EC, Wotherspoon A, Peckitt C, et al. Mismatch Repair Deficiency, Microsatellite Instability, and Survival: An Exploratory Analysis of the Medical Research Council Adjuvant Gastric Infusional Chemotherapy (MAGIC) Trial. JAMA Oncol 3: 1197-1203, 2017.

12. Evrard C, Tachon G, Randrian V, et al. Microsatellite instability: Diagnosis, heterogeneity, discordance, and clinical impact in colorectal cancer. Cancers (Basel) 11: 1567, 2019.

13. Chang L, Chang M, Chang HM, et al. Expending role of microsatellite instability in diagnosis and treatment of colorectal cancers. J Gastrointest Cancer 48: 305-313, 2017.

14. Theuer CP, Campbell BS, Peel DJ, et al. Microsatellite instability in Japanese vs European American patients with gastric cancer. Arch Surg 137: 960-965, 2002.

15. Mathiak M, Warneke VS, Behrens HM, et al. Clinicopathologic Characteristics of Microsatellite Instable Gastric Carcinomas Revisited: Urgent Need for Standardization. Appl Immunohistochem Mol Morphol 25: 12-24, 2017.

16. Buffart TE, Louw M, van Grieken NC, et al. Gastric cancers of Western European and African patients show different patterns of genomic instability. BMC Med Genomics 4: 7, 2011.

17. Polom K, Marrelli D, Pascale V, et al. High-risk and low-risk gastric cancer areas in Italy and its association with microsatellite instability. J Cancer Res Clin Oncol 142: 1817-24, 2016.

18. Cristescu R, Lee J, Nebozhyn M, et al. Molecular analysis of gastric cancer identifies subtypes associated with distinct clinical outcomes. Nat Med 21: 449-56, 2015.

19. Oki E, Kakeji $Y$, Zhao Y, et al. Chemosensitivity and survival in gastric cancer patients with microsatellite instability. Ann Surg Oncol 16: 2510-2515, 2009.

20. An JY, Kim H, Cheong JH, et al. Microsatellite instability in sporadic gastric cancer: its prognostic role and guidance for 5-FU based chemotherapy after RO resection. Int $\mathrm{J}$ Cancer 131: 505-511, 2012.

21. Hong SP, Min BS, Kim TI, et al. The differential impact of microsatellite instability as a marker of prognosis and tumour response between colon cancer and rectal cancer. Eur J Cancer 48: 1235-1243, 2012.

22. Kim DG, An JY, Kim H, et al. Clinical implications of microsatellite instability in early gastric cancer. J Gastric Cancer 19: 427-437, 2019.

23. Miceli R, An J, Di Bartolomeo M, et al. Prognostic impact of microsatellite instability in Asian gastric cancer patients enrolled in the ARTIST Trial. Oncology 97: 38-43, 2019.

24. Di Bartolomeo M, Morano F, Raimondi A, et al. Prognostic and predictive value of microsatellite instability, inflammatory reaction and PD-L1 in gastric cancer patients treated with either adjuvant 5-FU/LV or sequential FOLFIRI followed by cisplatin and docetaxel: A translational analysis from the ITACA-S Trial. Oncologist 24: e461-e468, 2019.

25. Choi YY, Kim H, Shin SJ, et al. Microsatellite instability and programmed cell death-ligand 1 expression in stage I//III gastric cancer: Post hoc analysis of the CLASSIC randomized controlled study. Ann Surg 270: 309-316, 2019.

\section{Correspondence: \\ Dr. Serdar ARICI}

Saglik Bilimleri Universitesi

Okmeydani Egitim ve Arastirma Hastanesi

Tibbi Onkoloji Bolumu

34384, Okmeydani

ISTANBUL / TURKEY

Tel: (+90-544) 7663930

e-mail: serdararici@hotmail.com

\section{ORCIDs:}

Serdar Arici

Selma Sengiz Erhan

Caglayan Geredeli

Muhammed Mustafa Atci

Saban Secmeler

Ruhper Cekin

Abdullah Sakin

Sener Cihan
0000-0003-2018-6554

0000-0001-8810-8806

0000-0002-3982-7465

0000-0002-1300-3695

0000-0001-8421-9234

0000-0002-7111-8482

0000-0003-2538-8569

0000-0002- 3960-7465 